\title{
Histopathologic and flow-cytometric analysis of neoplastic and benign "background" tissue in breast carcinoma resections *
}

\author{
Daniel W. Visscher ${ }^{* *}$, Debra S. Gingrich, Carmen Leon-Armin, Pamela Tabaczka and \\ John D. Crissman \\ Department of Pathology at Harper Hospital, Karmanos Cancer Institute, Wayne State University \\ School of Medicine, Detroit, MI, USA
}

Received 6 June 1998

Revised 27 August 1998

Accepted 27 August 1998

\begin{abstract}
Two-color, multiparametric synthesis phase fraction (SPF) analysis of cytokeratin-labeled epithelial cells was flow cytometrically performed on both benign ( $\mathrm{SPFb}$ ) and malignant tissue samples (if available, SPFt) from 132 mastectomy/lumpectomy specimens. These data were then correlated with clinicopathologic features, including (1) tumor differentiation, (2) the proportion of tumor comprised of duct carcinoma-in situ (DCIS), and (3) the histology of accompanying benign breast tissue, classified by predominant microscopic pattern as intact, normal terminal duct lobular units (NTDLU, 34\% of cases), atrophic (AT, 33\% of cases), proliferative fibrocystic (PFC, 26\% of cases), and non-proliferative fibrocystic (NPFC, 7\% of cases). SPFt was inversely correlated with extent of DCIS (DCIS $=0-20 \%$ tumor volume $-12.7 \%$ mean SPFt, vs. DCIS $>20 \%$ tumor volume $-6.4 \%$ mean SPFt, $p=0.001$ ). SPFt also correlated with the histology of background benign breast tissue (NTDLU - 14.8\% mean SPFt vs. AT $-6.9 \%$ mean SPFt vs. PFC $-12.7 \%$ mean SPFt, $p=0.05$ ) but it did not correlate with patient age or SPFb (overall mean $=0.73 \%)$. SPFb was correlated with patient age $(>56 \mathrm{yr}-0.59 \%$ mean SPFb vs. $<56 \mathrm{yr}-$ $0.84 \%$ mean SPFb, $p=0.02$ ), with background histology (NTDLU $-1.1 \%$ mean SPFb vs. AT $-0.43 \%$ mean SPFb vs. PFC $-0.70 \%$ mean SPFb, $p<0.02$ ) and with the grade of the neoplasm (well/moderate $-0.58 \%$ mean vs. poorly differentiated $-0.85 \%$ mean, $p=0.04)$. Patients having a background of PFC were significantly older than patients with a background of NTDLU (45.2 yr vs. $60.2 \mathrm{yr}, p=0.01)$.

We conclude: (1) breast carcinomas arising from a background of more actively cycling pre-involutional or proliferative fibrocystic epithelium have a greater proliferative fraction than tumors arising from atrophic epithelium, implying that the differentiation status of target cells may impact the effect(s) of tumorigenic events; (2) PFC may represent delayed, abnormal or interrupted involution rather than a hyperproliferative state relative to NTDLU, suggesting that it facilitates neoplasia by extending the period of exposure to promoter agents such as endogenous hormones, and (3) lower SPFt in breast neoplasia with more abundant "residual" DCIS may reflect a lengthier pre-invasive disease interval due to intrinsically less aggressive phenotype.
\end{abstract}

Keywords: Synthesis phase fraction, fibrocystic disease, flow cytometry, breast carcinoma

\section{Introduction}

Differentiation status or pathologic alterations involving benign breast tissue are widely held to be important biological factors impacting the genesis and phenotype of breast carcinoma. Probably the

\footnotetext{
*Presented at the 1998 USCAP Annual Meeting, Boston, MA, USA, 28 February-6 March 1998.

*** Corresponding author: Daniel W. Visscher, M.D., Department of Pathology, Harper Hospital, 3990 John R., Detroit, MI 48201, USA. Fax: +1 3137459299.
} 
best known example of this association is the greater frequency of hormone receptor positive neoplasms which arise in older, post-menopausal patients. Breast tissues in such cases typically demonstrate a background of atrophic lobular epithelium, due to age-related involution. A second example is the increased risk of breast carcinoma conferred by the presence of proliferative fibrocystic (PFC) change, most patterns of which are generally considered to represent forms of hyperplasia. Some fibrocystic lesions, such as atypical hyperplasia, exhibit pathologic features more in keeping with intra-epithelial neoplasia (i.e., dysplasia). Not all breast carcinomas, however, develop from a background of fibrocystic changes [1]. From a purely histopathologic stand point, in fact, breast carcinoma is almost unique among human tumor systems for the sheer variety of "background" tissue patterns from which it may arise: these include "normal" terminal duct lobular unit (TDLU) parenchyma, atrophic TDLU parenchyma, a variety of proliferative or non-proliferative fibrocystic changes (PFC/NPFC), or (most commonly) admixtures of "normal", atrophic and/or fibrocystic. Although relatively few authors have systematically evaluated human breast carcinoma in the context of "background" breast histology, the above considerations would imply that such analyses may reveal clues about the underlying basis of breast carcinogenesis and clinicopathologic heterogeneity.

Using suspensions of dissociated cells with intact cytoplasm, this study employs "two-color" multiparametric flow cytometry in order to compare cell cycle analyses from samples of both neoplasm and accompanying benign tissues derived from lumpectomy/mastectomy specimens. Multiparametric DNA analysis employs computer "gating" to select for, and thereby restrict analysis to cells which have been "labelled" with a fluorescent-tagged antibody; herein we employed cytokeratin, a cytoplasmic intermediate filament specific for epithelial cells. In this manner, the DNA and cell cycle calculations were limited to the populations of interest, excluding stromal/inflammatory contaminants. This is important due to the fact that at least $50 \%$ of cells in benign (or malignant) breast tissue are non-epithelial. These events would contaminate DNA histograms and interfere with cell cycle calculations. Epithelial cell specific synthesis phase fractions from both neoplastic and benign breast tissues were then correlated with clinicopathologic features, especially histologic patterns of "background" benign tissues. In view of the issues noted previously, it was our hypothesis that benign "background" epithelial cell proliferation and histology are variables which may be reflected in the clinicopathologic patterns of human breast neoplasia.

\section{Materials and methods}

\subsection{Case selection and pathologic assessment}

Lumpectomy and/or mastectomy specimens $(N=150)$ submitted to the Department of Pathology at Harper Hospital, Detroit, Michigan (years 1996-97 inclusive) were obtained and evaluated in a prospective manner. Mean patient age was 55.8 years. Following careful dissection and gross examination, representative $1 \mathrm{~cm}$ in dimension tissue slices of both benign and malignant tissue (if available) were partitioned from fresh specimens and placed in RPMI transport medium. Grossly benign samples, when possible, were obtained from a quadrant not involved by the neoplasm and were free of focal lesions such as cysts, fibroadenomas, papillomas or prior biopsy tracts. A "mirror image" slice of each benign sample was fixed in formalin and utilized to prepare a paraffin-embedded, hematoxylin and eosin stained tissue section. This served not only to confirm the absence of malignant neoplasm but also to characterize the microscopic features of the benign sample. This was performed by one author (DV). Each benign sample was classified histologically into one of four groups employing criteria as follows: 
(1) normal (intact, non-atrophic terminal duct lobular units comprising at least $80 \%$ of the sampled epithelium),

(2) atrophic (small, involuted terminal duct lobular units, or ducts without acini, comprising at least $80 \%$ of the sampled epithelium),

(3) non-proliferative fibrocystic (apocrine metaplasia, lobular distortion without hyperplasia with or without fibrosis involving at least $50 \%$ of the section), or

(4) proliferative fibrocystic (any form of epithelial hyperplasia, including adenosis, duct hyperplasia or atypical hyperplasia involving at least one third of sampled epithelium).

With the exception of (3) and (4) these categories are mutually exclusive. If a sample fulfilled criteria for both (3) and (4), it was arbitrarily classified as (4). As a further histologic control, Papanicolaou-stained cytospin preparations of all dissociated cell suspensions were microscopically examined for the presence (or absence) of benign/malignant epithelial cells. Cases in which "benign" samples contained any histologic or cytologic evidence of malignant cells were excluded $(N=18)$ resulting in a sample size of 132.

All routinely-obtained histologic slides from the main specimen (and prior biopsies, if available, $N=83$ ) were examined in order to characterize both the neoplasm and the accompanying benign breast parenchyma. Each neoplasm was evaluated for differentiation (well, moderate, poor) as well as the approximate volume (expressed as the percent of total volume) of in situ carcinoma. Finally, from routinely obtained sections derived from biopsy/lumpectomy/mastectomy specimens, "background" benign tissue from each case was evaluated for its approximate composition (expressed as a percentage) of normal vs. atrophic vs. non-proliferative or proliferative fibrocystic areas, and then classified using criteria as previously described. All cases had at least one random section obtained from quadrants/areas not involved by neoplasm (minimum number of sections containing benign tissue $=8$, mean $=12$ ).

\subsection{Multiparametric cell cycle analysis}

Intact single cell suspensions were obtained mechanically, by scraping the surfaces of tissue specimens with a scalpel blade. This was followed by centrifugation, re-suspension and fixation with cold ethanol. Specific details of our 2-color, multiparametric method of cell cycle analysis have been previously published [4]. Briefly, an aliquot of suspension is dual-labelled with both propidium iodide as well as a FITC-conjugated anti-cytokeratin antibody (CAM 5.2, Becton-Dickenson, Mountain View, CA). Cells of epithelial derivation could thereby be specifically identified during data acquisition with the use of specimen and (non-immune) antibody-negative control green fluorescence (FL 1) histograms as illustrated in Fig. 1. At least 5000 cytokeratin-positive events were obtained from each sample. The DNA histograms resulting from this technique were superior, with minimal debris and optimal coefficients of variation (mean $=2.5 \%$, all were $<4 \%$ ). Cell cycle calculations were performed using an automated software program (CellFit, Becton-Dickenson) which contains a pulse-height aggregate discrimination module.

\subsection{Data analysis}

Patient ages were obtained from surgical pathology reports. Comparisons between sets of parameters (i.e., $t$-tests) were performed using commercially-available, statistical software on a personal computer. 

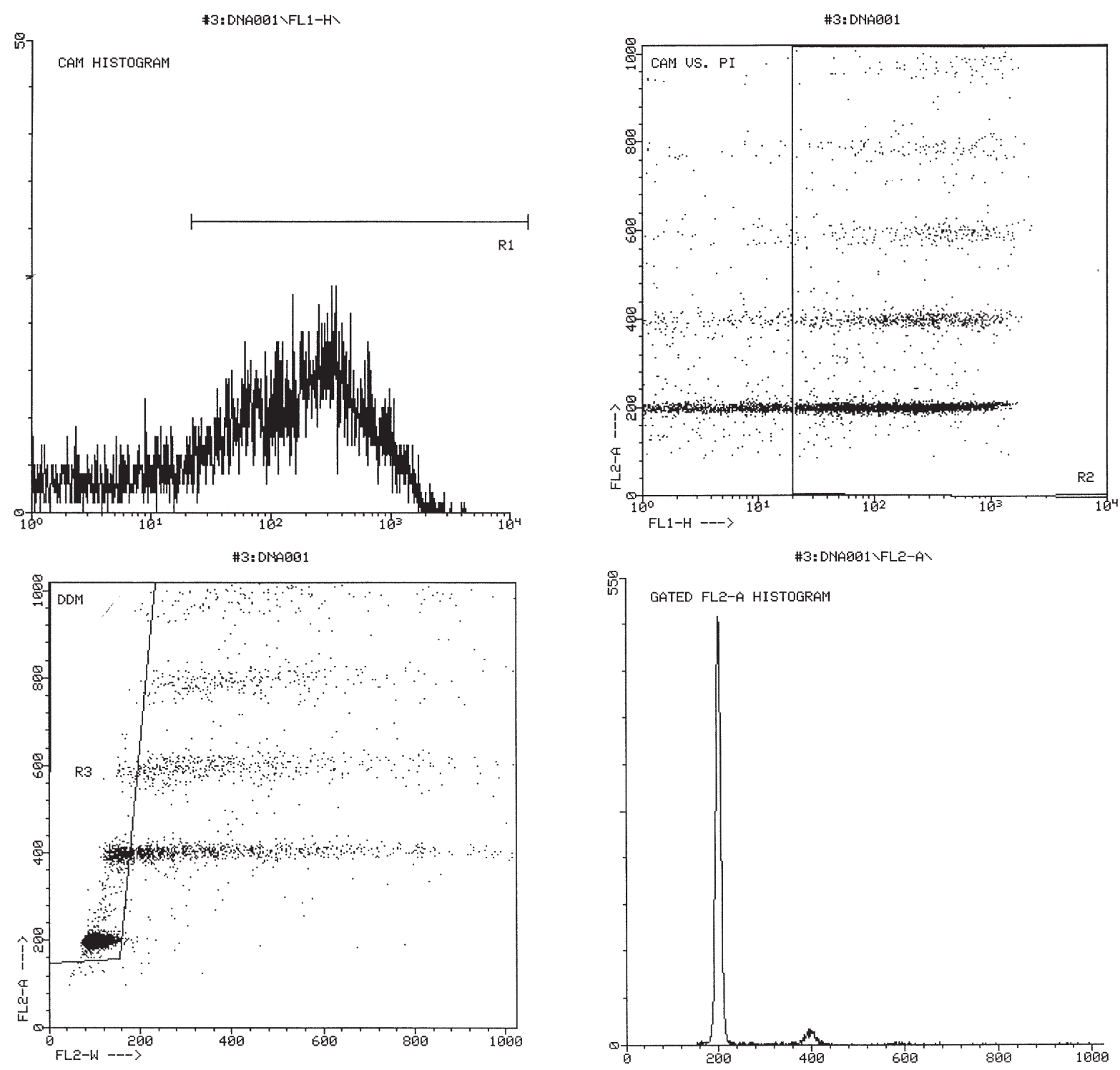

Fig. 1. Top left: fluorescence-1 (green fluorescence) histogram exhibiting a population of cytokeratin positive cells, designated by R1. Top right: histogram of DNA content (vertical axis) versus cytokeratin fluorescence intensity (horizontal axis). The $\mathrm{R} 1$ gate is illustrated by the vertical line. Note the presence of populations corresponding to aggregates. Lower left: gate R3 corresponding to the aggregate subtraction component of the doublet discrimination module. Lower right: cytokeratin gated DNA histogram, demonstrating a unimodal G0G1 population with low coefficient of variation and minimal debris or aggregates.

\section{Results}

\subsection{Neoplasm proliferation (SPFt) and clinicopathologic parameters (Table 1)}

The mean synthesis phase fraction of tumor cells (11.2\%) was more than ten fold greater than mean SPF for benign breast tissues $(0.73 \%)$, although both were characterized by considerable range of variation (SPFt: 2-42\%, SPFb: 0.1-3.8\%). There was no apparent relationship between patient age and degree 
Table 1

\begin{tabular}{|c|c|c|}
\hline Parameter $(N)$ & Mean SPFt (SD) & $p$ value \\
\hline \multicolumn{3}{|l|}{ Age } \\
\hline$<56$ yr (32) & $11.0(10.3)$ & NS \\
\hline$>56$ yr (31) & $11.4(8.7)$ & \\
\hline \multicolumn{3}{|l|}{ Tumor grade } \\
\hline I (6) & $1.2(0.77)$ & $<0.001$ \\
\hline II (24) & $7.2(5.5)$ & \\
\hline II (31) & $16.1(9.4)$ & \\
\hline \multicolumn{3}{|l|}{$\%$ DCIS in tumor } \\
\hline 0-20\% (43) & 12.7 & 0.007 \\
\hline$>20 \%$ & 6.4 & \\
\hline \multicolumn{3}{|l|}{ Benign histology } \\
\hline (1) normal (20) & $14.8(9.7)$ & 0.001 (1) vs. (2)) \\
\hline (2) atrophic (17) & $6.9(4.4)$ & 0.05 (2) vs. (4)) \\
\hline (3) $\mathrm{NPFCD}$ (5) & $6.3(4.2)$ & \\
\hline (4) PFCD (18) & $12.7(9.5)$ & \\
\hline \multicolumn{3}{|l|}{$\%$ Normal TDLU } \\
\hline (1) $0-30(31)$ & $9.0(7.7)$ & \\
\hline (2) $>30$ (28) & 13.7 (9.9) & 0.05 \\
\hline \multicolumn{3}{|l|}{$\%$ PFCD } \\
\hline (1) $0(20)$ & $14.3(11.9)$ & \\
\hline (2) $1-20(25)$ & $8.5(7.3)$ & NS \\
\hline (3) $>20$ (14) & $11.8(9.2)$ & \\
\hline
\end{tabular}

of tumor cell proliferation whether evaluated as mean SPFt in groups of patients above vs below mean age (Table 1) or by age in decades (i.e., 30-40 yr, 41-50 yr, etc.,..., data not shown). SPFt was strongly correlated with tumor differentiation; the mean SPF of poorly-differentiated neoplasms (16.1\%) was more than double that of moderately-differentiated lesions $(7.2, p<0.001 \%)$. SPFt was also significantly greater ( $12.7 \%$ mean) among those neoplasms which were characterized by limited DCIS $(<20 \%$ of tumor volume) as opposed to cases (6.4\% mean SPFt) with extensive DCIS ( $>20 \%$ tumor volume) $(p=0.001)$. This correlation, though, may largely reflect a significant association between tumor differentiation and the proportion of DCIS. The mean percent tumor volume comprised of DCIS in poorly differentiated carcinomas was 9.7 , vs. $27.1 \%$ for well or moderately differentiated tumors $(p=0.001)$.

Benign tissue samples submitted for FCM were about equally divided between histologically "normal" TDLU, atrophic breast tissue (32 and 33\%, respectively) and fibrocystic change (34\%). The mean SPFt was greatest in tumors characterized by a (predominant) background of "normal" (that is, non-atrophic and non-fibrocystic) tissue. Cases in which the benign sample submitted for flow cytometric cell cycle analysis was histologically "normal" had a mean SPTt of $14.8 \%$. This is significantly greater than mean SPTt $(6.9 \%)$ of cases in which the benign tissue submitted for cell cycle analysis was histologically atrophic $(p=0.001)$, but it was not statistically different from cases in which the benign sample exhibited proliferative fibrocystic change (12.7\%). The numbers of samples comprised of non-proliferative fibrocystic changes was insufficient for meaningful statistical analysis.

Since samples of benign tissue submitted for flow cytometric analysis were obtained prospectively and thus not necessarily representative of overall "background" breast histology, these results are potentially vulnerable to selection artifacts. However, similar correlations between SPFt and predominant 
"background" histology (i.e., as assessed by reviewing all histologic sections) were also apparent. The mean SPFt in tumors accompanied by predominantly "normal" breast tissue was $16.6 \%$, vs. $7.9 \%$ for cases with a predominantly atrophic background $(p=0.03)$ and $15.5 \%$ for cases with a fibrocystic background. The sample of benign tissue harvested for cell cycle analysis was usually, but not always, morphologically representative of the overall background epithelium; $76 \%$ of the samples reflected the predominant histology in routinely obtained sections. This degree of concordance derives largely from the homogeneity of lumpectomy/mastectomy tissues characterized by "normal" or "atrophic" TDLU epithelium. Most discrepancies, accordingly, reflected the focal nature of fibrocystic change and its intimate admixture with "normal" and/or "atrophic" epithelium. Proliferative fibrocystic alterations were, overall, observed in $72 \%$ of lumpectomy/mastectomy specimens. However, it comprised $0-10 \%$ of benign epithelium in $32 \%, 10-30 \%$ of benign epithelium in $15 \%$ and $>30 \%$ of epithelium in only $24 \%$ of cases.

\subsection{Correlations between SPFb and clinicopathologic parameters (Table 2)}

Benign epithelial cell proliferation was weakly, albeit significantly, correlated with patient age; mean $\mathrm{SPFb}$ in patients less than 56 yrs was $0.84 \%$, vs. $0.59 \%$ for patients greater than $56 \mathrm{yrs}(p=0.02)$. SPFb was more strongly associated, however, with the histology of benign epithelium. The mean SPFb in samples composed of non-atrophic "normal" tubular duct lobular units was $1.1 \%$, vs. only $0.43 \%$ for samples showing atrophic breast tissue $(p=0.001)$. Proliferative fibrocystic tissues, interestingly, were characterized by a mean SPF $(0.70 \%)$ which was lower, but not significantly different from that of "normal" breast tissue. SPFb failed to correlate with the extent of proliferative disease in lumpectomy/mastectomy tissues. It was $0.72 \%$ for cases in which benign epithelium was comprised of an estimated 1-20\% PFCD, vs. $0.64 \%$ in cases with $>20 \%$ PFCD. In contrast, SFPb was significantly correlated with the extent of nonatrophic "normal" epithelium present $(0-20 \%=0.59 \%$ vs. $21-40 \%=0.64 \%$ vs. $41-60 \%=0.75 \%$ vs. $>60 \%=1.1 \%, p=0.02$ ).

The observed relationships between SPFb and patient age or background histology in large part reflected the strong association between patient age and background histology. The mean age of patients

Table 2

Correlations between benign breast epithelial cell proliferation (SPFb) and clinicopathologic parameters

\begin{tabular}{lcc}
\hline Parameter $(N)$ & Mean SPFb (SD) & $p$ value \\
\hline Age & $0.593(0.384)$ & 0.05 \\
$<56(60)$ & $0.843(0.754)$ & \\
$>56(72)$ & & \\
Histology & $1.067(0.802)$ & (1) vs. (2) 0.000 \\
(1) normal (40) & $0.429(0.316)$ & (1) vs. (3) 0.0015 \\
(2) atrophic (41) & $0.580(0.220)$ & (1) vs. (4) 0.016 \\
(3) NPFCD (10) & $0.700(0.430)$ & \\
(4) PFCD (32) & & 0.038 \\
Tumor grade & $0.583(0.394)$ & \\
well/moderate (41) & \\
poor (46) & $0.854(0.763)$ & NS \\
PFCD extent & & \\
$0(39)$ & $0.831(0.661)$ & \\
1-20\% (53) & $0.717(0.747)$ & \\
$>20 \%(51)$ & $0.616(0.312)$ & \\
\hline
\end{tabular}


in which the benign sample submitted for cell cycle analysis consisted of "normal" tissue was $45.2 \mathrm{yrs}$, compared to 62.4 yrs for patients with "atrophic" samples $(p<0.001)$. Despite having a mean SPFb approaching that of samples with "normal" tissue though, the mean age of patients having proliferative fibrocystic tissue samples (60.2 yrs) was not significantly different from those with atrophic tissues. As may be anticipated, the amount of morphologically "normal" TDLU parenchyma declined with advancing patient age; it comprised $63 \%$ of the total epithelium for patients $0-44$ yrs, vs. $44 \%$ for patients $45-54$ yrs, $30 \%$ for patients 55-66 yrs and 17\% for patients $67-91$ yrs $(p<0.01)$. An analogous, statistically significant reciprocal association was observed between patient age and the extent of atrophic epithelium ( $p=0.001)$. Due in large part to the focal nature of proliferative fibrocystic alterations however, it was not possible to identify a straight forward relationship between patient age and the extent of PFCD within lumpectomy/mastectomy tissues. The mean percent of epithelium comprising PFCD in 24-44 yr old patients was $8.4 \%$, vs. $18 \%$ for patients $45-54$ yrs and $21 \%$ for patients $55-66$ yr. In 67-91 year olds, though, \%PFCD dropped to $14.3 \%$. The complex interaction between age, histology and SPFb is further highlighted by comparing SPFb from groups having similar histology but differing age. The mean SPFb in samples composed of "normal" TDLU from patients aged 0-44 yrs $(N=20)$ was $1.2 \%$, vs. $0.94 \%$ for patients $45 \mathrm{yrs}$ or older $(p=\mathrm{NS}$ ). Age-associated differences in proliferation were also lacking in samples containing PFCD (mean SPFb 0-60 yrs $=0.66 \%$, vs. $0.74 \%$ for patients $\geqslant 61 \mathrm{yrs}$ ).

Finally SPFb was significantly greater $(0.85 \%$ mean $)$ in patients with poorly differentiated, vs. well or moderately differentiated neoplasms $(0.55 \%$ mean, $p=0.04)$. However, there was no statistically significant overall correlation between $\mathrm{SPFb}$ and $\mathrm{SPFt}$.

\section{Discussion}

Correlations between SPFt and breast tumor differentiation have been previously reported by numerous authors. This study is the first, of which we are aware, to observe an association between the presence of extensive DCIS and low SPFt. This relationship may seem paradoxical in view of the reported proclivity of tumors with extensive in situ components to recur [2]. Such recurrences, however, develop in residual breast parenchyma (i.e., in patients treated with conservative surgery) and they do not necessarily forbode a disease-associated mortality. Indeed, the risk for local relapse in such cases seems to be conferred primarily on the basis of occult deposits of residual un-resected tumor cells. Some authors, in fact, have shown that the incidence of axillary nodal metastasis varies inversely with DCIS extent (i.e., among invasive lesions of similar size) and thus lesions with extensive in situ neoplasm may constitute in inherently less aggressive disease subset [3], at least with respect to metastatic potential. To the extent that SPF reflects tumor aggressiveness, our study would seem to support that view. This interpretation would be further supported by the observed significant correlation in this study between extensive DCIS and a well or moderately (i.e., vs. a poorly) differentiated invasive neoplastic component. Thus, the tendency for widespread in situ neoplasm to develop prior to, or concurrent with, invasive growth would appear to reflect a generally less aggressive form of breast neoplasia.

A noteworthy finding of this study was the lack of significant difference between the overall mean $\mathrm{SPFb}$ of "normal" vs. "proliferative fibrocystic" tissues. Our laboratory has reported similar findings using samples derived from benign breast biopsies [4]. Importantly, fibrocystic aliquots in this study were derived from patients who were significantly older than patients having histologically "normal" (i.e., non-atrophic) tissue samples. (They were not different, in fact, from the mean age of patients having atrophic samples.) Thus, our data imply that proliferative fibrocystic change represents not so much a 
state of hyper-proliferation vis-à-vis "normal", but rather a level of proliferation which is inappropriate for patient age, and hence the expected degree of atrophy/involution. In this sense, then, fibrocystic change may be viewed as a manifestation of delayed, inappropriate or interrupted atrophy. It is important to note that "normal", fibrocystic and even atrophic breast tissues are characterized by a high level of inter-patient variation with respect to SPF, as demonstrated by the large SD's in each category. The extent to which this is a reflection of heterogeneous tissue composition within individual breast tissues, temporal variation (e.g., as with hormonal cycling) or simply intrinsic inter-patient variability remains unclear.

Retention of pre-involutional cell cycling characteristics in fibrocystic tissues might have relevance to the observation that mean neoplastic cell SPF was similar in groups of cases having normal TDLU or proliferative fibrocystic backgrounds (14.8 and 12.7\%, respectively); both were greater than in cases with a predominantly atrophic background (6.9\%). It may also account for the greater mean SPFb among poorly-differentiated carcinomas vs. well and moderately differentiated carcinomas $(0.85 \%$ vs. $0.58 \%)$, since $74 \%$ of tumors having a background of "normal" TDLU were poorly-differentiated, compared to $42 \%$ of tumors having a predominantly atrophic background. Although an association between neoplastic and "background" epithelial phenotypes, overall, thus seems established, the nature of this relationship is speculative. One might hypothesize that more actively cycling benign populations have a "head start", so that genetic alterations leading to hyper-proliferation and malignant neoplasia are imposed on cells which are intrinsically less restricted with respect to cell cycle progression, leading ultimately to a rapidly-proliferating, poorly differentiated neoplastic phenotype. Alternatively, actively cycling benign populations may be more susceptible to mutational influences, thereby resulting in a relatively greater degree of genetic instability, by virtue of mitotic errors, and hence less differentiated phenotype. The latter hypothesis is supported by the well established correlation between elevated proliferative fraction, DNA aneuploidy and high grade in breast neoplasia.

With respect to fibrocystic changes, the data are not incompatible with the notion that "proliferative" alterations may predispose to malignancy by virtue of abnormally retained sensitivity to substances such as estrogen, that stimulate cell cycling in tissues which would normally be atrophic. Proliferative fibrocystic lesions have been shown to have upregulated expression of hormone receptors [5]. This model would account for the lack of hyper-proliferation vis-à-vis "normal" TDLU epithelium and also for the well-documented lengthy interval between the diagnosis of fibrocystic changes and appearance of subsequent malignant neoplasia. A competing hypothesis is that the presence of PFCD reflects the consequences of exposure to carcinogenic stimuli, and thus represents a morphologic hallmark of "partial" malignant transformation akin to colorectal adenoma. Such lesions would be expected to demonstrate unequivocal elevation of proliferative fraction. Although our study did not reveal increased SPF in proliferative fibrocystic lesions relative to "normal" lobules, we did not compare proliferative fibrocystic tissue to "normal" or atrophic lobules within the same breast. Nor did we evaluate a series of patients with benign breasts. We cannot fully discount the possibility that PFC may, in at least some cases, arise from atrophic TDLU epithelium. Thus, subtle elevations in proliferative fraction were not fully excluded.

The measurable frequency of cases without any significant background of PFC would imply, moreover, that not all breast carcinomas develop through, or concurrent with, such changes. This finding is under emphasized in generally accepted current models of breast carcinogenesis. These cases may represent distinct subsets of breast neoplasia having a different pathogenesis, possibly involving divergent carcinogenic factors and/or genetic pathology. Further studies, in our opinion, which explore the relationship between neoplastic features and precursor tissue alterations may thus prove useful in refining our understanding of human breast carcinoma. 


\section{References}

[1] E.R. Fisher, S. Anderson, C. Redmond and B. Fisher, Pathologic findings from the national surgical adjuvant breast project protocol B-06: 10-year pathologic and clinical prognostic discriminants, Cancer 71 (1993), 2507-2514.

[2] S.J. Schnitt, A. Abner, R. Gelman, J.L. Connolly, A. Recht, R.B. Duda, T.J. Eberlein, K. Mayzel, B. Silver and J.R. Harris, The relationship between microscopic margins of resection and the risk of local recurrence in patients with breast cancer treated with breast-conserving surgery and radiation therapy, Cancer 74 (1994), 1746-1751.

[3] S.G. Silverberg and A.R. Chitale, Assessment of significance of proportions of intraductal and infiltrating tumor growth in ductal carcinoma of the breast, Cancer 32 (1973), 830-837.

[4] D.W. Visscher, D.S. Gingrich, J. Buckley, P. Tabaczka and J.D. Crissman, Cell cycle analysis of normal, atrophic and hyperplastic breast epithelium using two-color multiparametric flow cytometry, Anal. Cell. Pathol. 12 (1996), 115-124.

[5] D.W. Visscher, N. Padiyar, D. Long and P. Tabaczka, Immunohistologic analysis of estrogen receptor expression in breast carcinoma precursor lesions, The Breast J. (in press). 


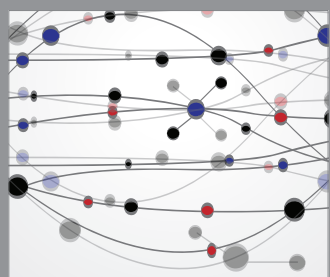

The Scientific World Journal
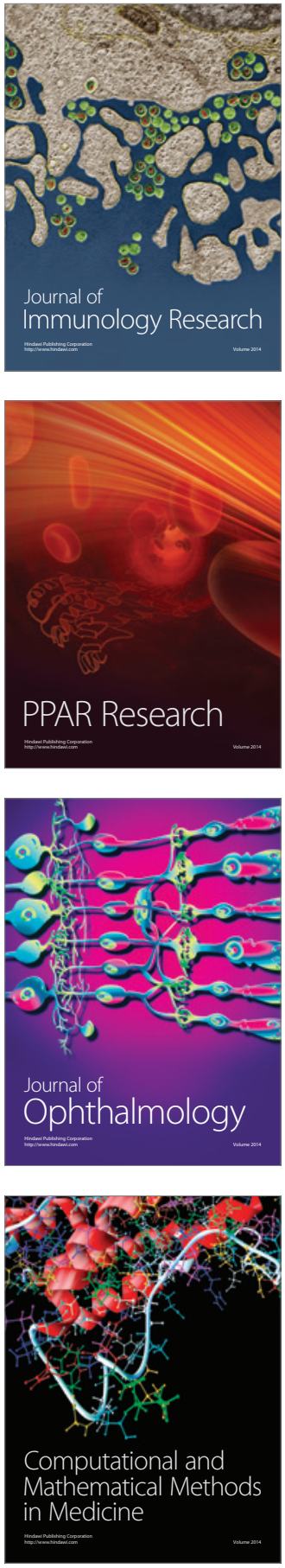

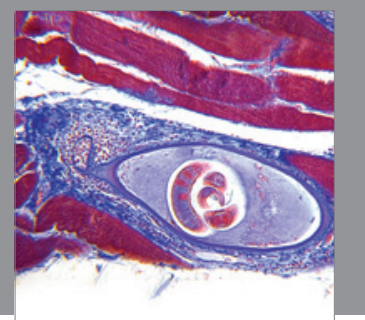

Gastroenterology

Research and Practice
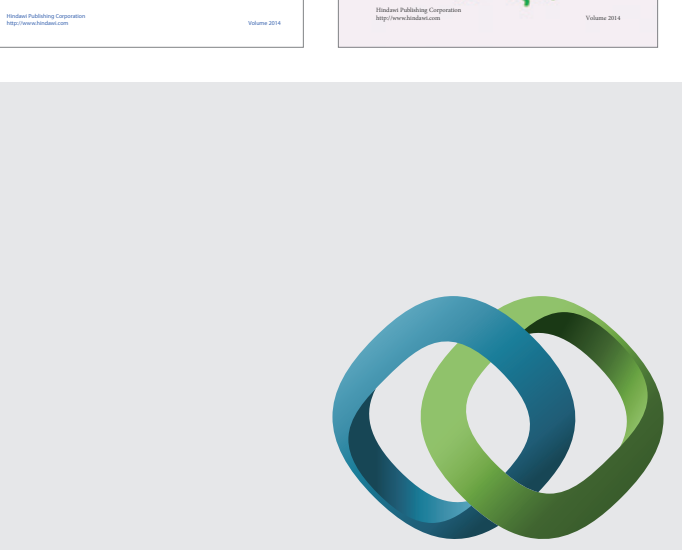

\section{Hindawi}

Submit your manuscripts at

http://www.hindawi.com
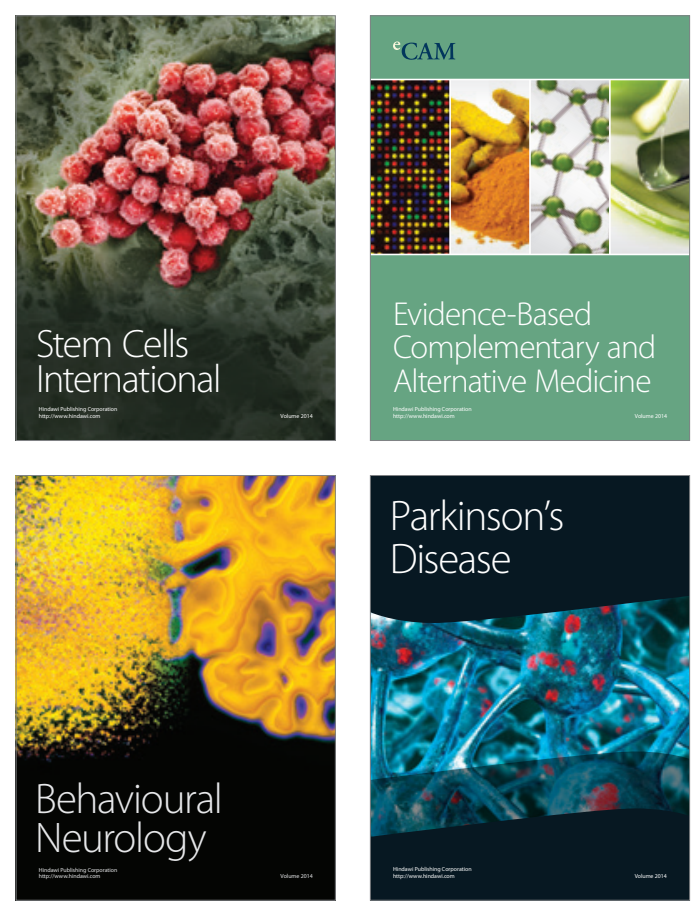

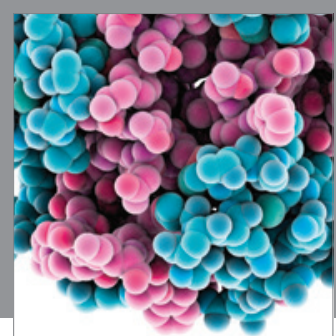

Journal of
Diabetes Research

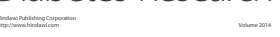

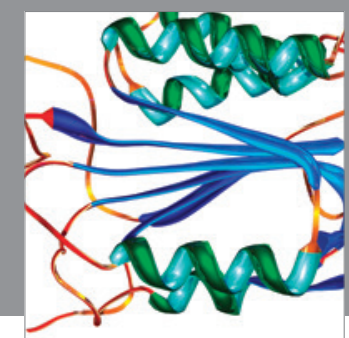

Disease Markers
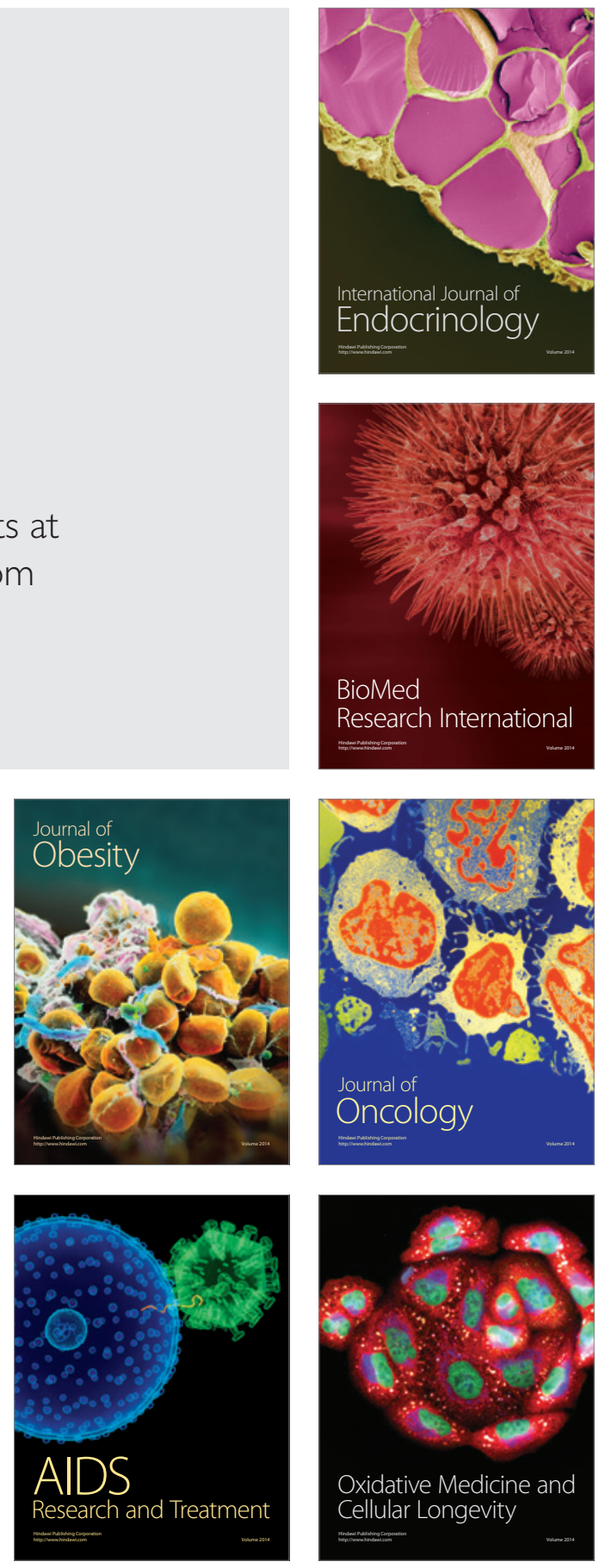